\title{
Idiopathic Epiretinal Membrane in Young Patient: A Rare Case Presentation
}

Raba Thapa,' Govinda Paudyal'

'Tilganga Institute of Ophthalmology, Gaushala, Kathmandu, Nepal.

\begin{abstract}
Epiretinal membrane is avascular fibrous tissue which is adhered to the internal layers of retina in the macular area. Patient may be asymptomatic or present with blurring of vision and metamorphopsia. The idiopathic Epiretinal Membrane is more often seen in elderly but it could be present in young patients in association with other vitreo-retinal diseases and following intraocular surgery. The idiopathic Eperetinal Membrane in young patients are rare. Here we present a thirty one year healthy women presented with idiopathic Epiretinal Membrane who gained full visual recovery following the pars plana vitrectomy, membrane peeling and gas for its rarity.
\end{abstract}

Keywords: epiretinal membrane; idiopathic; young women.

\section{INTRODUCTION}

Epiretinal membrane (ERM) is a common retinal problem especially in the elderly. The population based studies had shown the prevalence of ERM of $11.8 \%$ in Beaver Dam Eye Study, ${ }^{1}$ 7\% in Blue Mountain Eye Study (BMES), ${ }^{2}$ and $7.9 \%$ in Asian Malaysia population. ${ }^{3}$ The association of ERM with age is clear and more than $90 \%$ of the patients are older than 50 years. Diabetes mellitus, cataract surgery, and retinal vein occlusion are the common associations with ERM. Idiopathic ERM is rare presentations at young age. ${ }^{2}$ Mild visual disturbance with metamorphopsia are often the presenting complains with ERM. Pars-plana vitrectomy with membrane peeling is the definitive way of managing ERM resulting in improvement of vision and metamorphopsia.

\section{CASE REPORT}

Thirty one year female presented at Tilganga Institute of Ophthalmology, a tertiary eye hospital in Nepal with chief complain of blurring of vision associated with metamorphopsia of two weeks duration in the right eye (RE) on June 2010. She had no other ocular problems including the ocular trauma and surgery. She had no ocular complains in her left eye (LE), and also no significant systemic problems. Her past ocular problems were unremarkable.

Her presenting vision in RE was $6 / 36$, that was improved with best correction (BCVA) to 6/24 (-0.50 $\left.\times 90^{\circ}\right)$ and BCVA in LE was 6/6 $\left(-0.25 \times 90^{\circ}\right)$

Anterior segment evaluation was done with the Haag Streit slit lamp and posterior segment evaluation was done with the help of 900 and 20D lens. Anterior segment findings were unremarkable in both the eyes. On posterior segment evaluation, both the core and peripheral vitreous was clear. Optic disc was normal in both the eyes. Macula shows epiretinal membrane with localized tractional macular detachment in the RE (Figure 1). Posterior, peripheral retina and retinal blood vessels revealed normal findings in both the eyes. Optical coherence tomography (OCT) also revealed epiretinal membrane in RE with central macular thickness (CMT) of 556 micron (Figure 2). Posterior

Correspondence: Dr. Raba Thapa, Tilganga Institute of Ophthalmology, Gaushala, Kathmandu, Nepal. Email: rabathapa@live.com, Phone: 977- 1- 4493775. 
segment examination of LE was normal with CMT 143 micron.

The intraocular pressure was $17 \mathrm{mmHg}$ in both eyes with applanation tonometer. Her blood pressure was normal $(100 / 70 \mathrm{mmHg})$. Her systemic investigations; complete blood counts, hemoglobin, blood sugar, VDRL/ TPHA/ HIV/HBsAg, chest X-ray revealed normal findings.

Because of her persistent visual problem, she was treated surgically after the informed consent with pars plana vitrectomy (23 Gauge), membrane peeling and perfluoropropane gas (C3F8 16\%). The complete vitreous separation and membrane peeling was assisted with the use of intravitreal triamsinolone. Intraoperatively, the core and peripheral vitreous was clear. Optic disc and peripheral retina was normal.

Patient was advised face down positioning for a week following the surgery along with topical steroid and antibiotics that were tapered and stopped within six weeks period. Her post-operative periods were uneventful without any rise of IOP and intraocular inflammations.

During her six weeks follow up, her presenting and BCVA was 6/18 and 6/9 respectively. The foveal reflex was dull but there was no residual membrane and rests of the findings were unremarkable. Her BCVA was 6/6 at her follow up visits of three months, six months, nine months and 12 months. At her last follow up, except the early posterior sub capsular cataract on the operated eye, the rest of the ocular findings was unremarkable with CMT of 246 micron (Figure 3). Patient was then advised to follow up in four months duration or earlier as and when felt necessary.
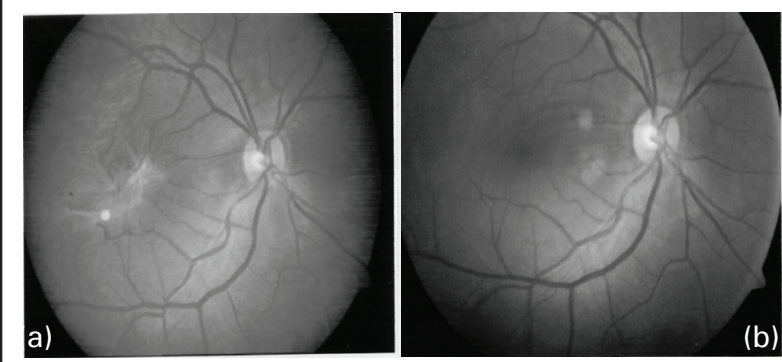

Figure 1. Fundus photo (a) before and (b) after surgery (1 year follow up).
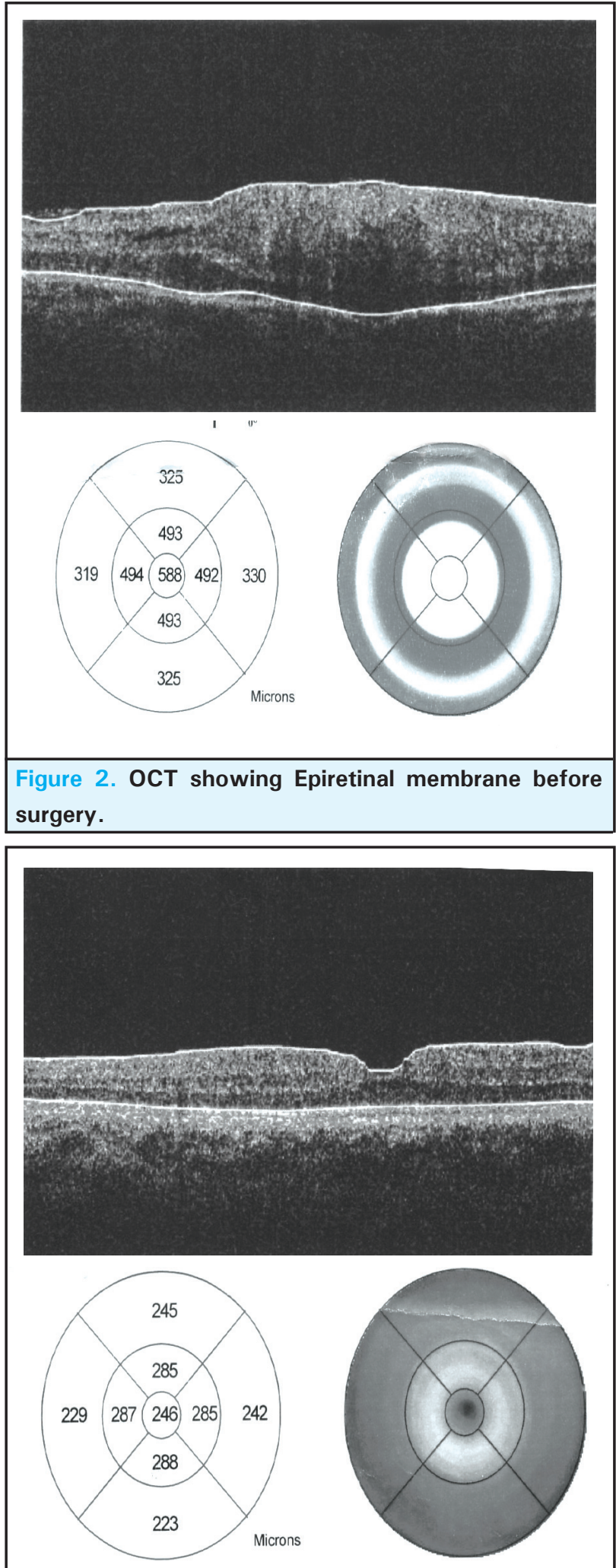

Figure 3. OCT showing macular thickness following vitrectomy and membrane peeling. 


\section{DISCUSSION}

Epiretinal membrane is more or less transparent avascular fibrous tissue which is adhered to the internal layers of retina in the macular area. ERM is often seen in elderly and more than $90 \%$ of cases are older than 50 years of age. The BMES reported ERM prevalence of $1.9 \%$ in persons younger than 60 years and $11.6 \%$ at the age of 70 to 79 years of age. The bilateral involvement was found in $31 \%$ of the cases. ${ }^{2}$ The presentation of young patients with idiopathic ERM is very rare entity although ERM due to secondary causes may present in any age groups. Cataract surgery $(16.8 \%)$, retinal vein occlusion $(16.1 \%)$, and diabetic retinopathy $(11 \%)$ were the common associations with ERM in BMES. ${ }^{2}$ The prevalence of ERM had been reported higher in females as compared to males as in our case. ${ }^{2,3}$

Visual acuity usually stabilizes after ERM formation and only $10 \%$ to $25 \%$ of patients lose one or two lines of vision when followed for a two year period. Occasionally, idiopathic ERM may induce slow and progressive vision loss throughout the years. Posterior vitreous detachment (PVD) is reported in up to $90 \%$ of patients with idiopathic ERM. ${ }^{4}$ Visual acuity may be poorer and risk of macular edema is higher with concurrent vitreo-macular traction (VMT). Our case had neither PVD nor VMT. ERM have been reported to resolve spontaneously, especially among younger patients in association with $\mathrm{PVD}^{5,6}$ but in our case, there was persistent ERM with metamorphopsia although vision was stable on three months follow up.

Most of the patients are asymptomatic and have normal vision. Only few patients present with blurring of vision and metamorphopsia. The aim of the surgery is to remove ERM causing significant vision loss. Surgery is usually reserved for those cases with visual deterioration, incapacitating metamorphopsia or monocular diplopia and those requiring improved $V A$ for the day to day work. ${ }^{7}$

There are many studies using various dyes like indocyanine green, tryphan blue to assist internal limiting membrane peeling by improving direct visualization of the membrane but with controversies of their toxic effects. ${ }^{8}$

Idiopathic ERM has better visual prognosis than the ERM resulted from retinal detachment. The better postoperative VA was demonstrated with macula sparing RD and presence of thin ERM. VA improved in 60 to $90 \%$ of cases and metamorphopsia resolved in 75 to $85 \%$ of cases in a series by de Bosters et al. ${ }^{9}$ In a similar report of idiopathic ERM, the factors associated with better VA included initial VA of 20/60 or better, short duration of symptoms of blurred vision, thin ERM and absence of tractional retinal detachment. Macular folds are reduced following surgery in $80 \%$ of cases and almost normal situation is achieved in $30 \%$ of the cases. ${ }^{10}$ In our case, patient had normal anatomical architecture of macula with full visual recovery following the vitrectomy and membrane peeling.

The most frequently reported complication was cataract, that was found in 40 to $80 \%$ of patients older than 60 years of age during the first year among the other complications like retinal phototoxicity, toxicity induced by vital stains and retinal tears, retinal detachment, recurrence of ERM and less frequently endophthalmitis. ${ }^{11}$ Our finding of early posterior subcapsular cataract in a year follow up was also consistent with their case series.

\section{CONCLUSIONS}

Although ERM is not a common retinal disorder in young healthy patients, timely surgical intervention with pars plana vitrectomy, and membrane peeling results in symptomatic improvement and good visual recovery.

\section{REFERENCES}

1. Klein R, Klein BE, Wang Q, Moss SE. The epidemiology of epiretinal membranes. Trans Am ophthalmol Soc. 1994;92:403-25.

2. Mitchell P, Smith W, Chey T, Wang JJ, Chang A. Prevalence and associations of epiretinal membranes. The Blue Mountains Eye Study, Australia. Ophthalmology. 1997; 104(6):1033-40.

3. Kawasaki R, Wang JJ, Mitchell P, et al. Racial difference in the prevalence of epiretinal membranes between Caucasians and Asians. Br J Ophthalmol. 2008;92(10):1320-4.

4. Sidd RJ, Fine SL, Owens SL, Patz A. Idiopathic preretinal gliosis. Am J Ophthalmol. 1982;94:44-8.
5. Sachdev N, Gupta V, Gupta A, Singh R. Spontaneous separation of idiopathic epiretinal membrane in a young patient. Int Ophthalmol. 2008;28(4):301-2.

6. Sakir M, Cekic O, Bayraktar S, Yilmaz OF. Spontaneous separation of epiretinal membrane in a child with Stargardt macular dystrophy. J Aapos. 2007;11(6):618-9.

7. Richard D, Bohm A, Current status of macula surgery;Indications and surgical possibilities. Ophthalmologe. 1999;96(9):622-34.

8. Kwok AK, Lai TY, Li WW, Woo DC, Chan NR. Indocyanine green-assisted peeling of the retinal internal limiting membrane. Ophthalmology. 2000:107(11):2010-4. 
9. de Bustors S, Rice TA, Michels RG, et al. Vitrectomy for macular pucker; Use after treatment of retinal tears or retinal detachment. Arch Ophthalmol. 1988;106(6):758-60.

10. De Bustrors S, Thompson JT, Michels RG, Rice TA, Glaser BM. Vitrectomy for idiopathic epiretinal membrane causing macular pucker. Br J Ophthalmol. 1988;72(9):692-5.
11. Hikichi T, Matsumoto N, Ohtsuka H, et al. Comparison of one year outcome following 23 and 20- gauge pars plana vitrectomy for preretinal membrane. Am J ophthalmol. 2009;147(4):639-49. 